\title{
The total fungal microbiome functionality
}

\author{
Robert Starke ${ }^{1}$, Petr Capek ${ }^{2}$, Daniel Morais ${ }^{1}$, Nico Jehmlich $^{3}$, Petr Baldrian $^{1}$
}

${ }^{1}$ Laboratory of Environmental Microbiology, Institute of Microbiology of the CAS, Praha, Czech Republic;

${ }^{2}$ Faculty of Science, University of South Bohemia, České Budějovice, Czech Republic; ${ }^{3}$ Molecular Systems Biology, Helmholtz-Center for Environmental Research, UFZ, Leipzig, Germany

Unveiling the relationship between taxonomy and function of the microbiome is crucial to determine its contribution to ecosystem functioning. However, while there is a considerable amount of information on microbial taxonomic diversity, our understanding of its relationship to functional diversity is still scarce. Here we used a meta-analysis of 377 completely annotated and taxonomically different fungal genomes to predict the total fungal microbiome functionality on Earth with all known functions from level 3 of KEGG Orthology using both parametric and non-parametric estimations. The unsaturated model described the accumulation of functions with increasing species richness significantly better ( $P$-value $<2.2 e-16)$ than the saturated model suggesting the presence of widespread and rare functions. Consistent with the parametric approach, the non-parametric Chao-1 estimator that assumes a maximum functional richness did not reach a plateau. Based on previous estimates of fungal species richness on Earth, we propagated the unsaturated model to predict a total fungal microbiome functionality of 42.4 million. Of those, only $0.06 \%$ are known today since the vast majority belongs to yet unknown rare functions. Logically, our approach not only highlighted the presence of two types of functions but pointed towards the necessity of novel and more sophisticated methods to unveil the entirety of functions to fully understand the involvement of the fungal microbiome in ecosystem functioning.

Ecosystem functioning ${ }^{1-4}$ is mediated by biochemical transformations performed by a community of microbes from every domain of life ${ }^{5}$; the microbiome. In every community, multiple organisms from different taxonomic groups can play similar if not identical roles in ecosystem functionality, the so-called

4 functional redundancy ${ }^{6}$. In fact, functional redundancy of certain functions was shown to be very high 5 with several hundreds to thousands of different taxa expressing the same function within one habitat ${ }^{7}$. These functions can be statistically inferred based upon homology to experimentally characterized genes and proteins in specific organisms to find orthologs in other organisms present in a given microbiome.

8 This so-called ortholog annotation is performed in KEGG Orthology (KO) ${ }^{8,9}$ that covers a wide range of 9 functional classes (level 1 of $\mathrm{KO}$ ) comprising cellular processes, environmental information processing, 
11 functions not included in the annotation of the two databases pathway or brite. Logically, however, the

12 bottleneck of describing microbiome functions is the low number of fully annotated specific organisms as they are limited to those that have undergone isolation and extensive characterization while the vast majority of organisms were not yet studied ${ }^{10,11}$ and the annotation is based on the similarity to the genomes of the very few studied model organisms. As a consequence, fungal microbiome functionality can be inferred based on the composition of the fungal microbiome and its relation to functional parameters ${ }^{12}$ as indicated by the frequent use of 18 S and ITS2 metabarcoding (5,990 publications with the keyword "18S sequencing" and 2,466 with "ITS2 sequencing" in PubMed as of October 3rd 2019). However, as the mere description of the fungal community structure cannot directly assess functionality despite its proficient use to find variables driving the abundance of certain taxa or across multiple taxa down to the species level in complex communities, recently shotgun sequencing of genes $(8,857)$ and transcripts (514) as well as metaproteomics (426) became more and more popular as a direct link between taxonomy and function. However, our understanding of functional diversity and its relationship to taxonomic diversity is still scarce. Here, we use both parametric and non-parametric estimators of functional richness to unveil the relationship between taxonomy and function in fungi with the aim to predict the total fungal microbiome functions on Earth. For this, we downloaded all taxonomically diverse and completely annotated fungal genomes $(n=377)$ from the integrated microbial genomes and microbiomes (IMG) of the Joint Genome Institute (JGI) on August $7^{\text {th }} 2019$ with taxonomic annotation on species level and functional annotation on level 3 of KO. The parametric estimation comprised of an accumulation curve (AC) ${ }^{13}$ of increasing functions with increasing species using 1,000 random permutations and its fit to a saturated and an unsaturated model of the Michaelis-Menten kinetics. Otherwise, Chao-1 for every $10 \%$ of species richness each with 20 replicates represented the nonparametric estimator. We hypothesized limited functionality with a plateau at high species richness and thus a better fit of the saturated model of the parametric approach and a stagnating Chao-1 estimator with increasing species richness.

The gene count of fungal phyla was not significantly (P-value $>0.5$ ) different than the average gene count of all fungi (Figure 1a). Admittedly, the sequenced genomes are heavily in favor of Ascomycota $(226=60.0 \%)$ and Basidiomycota $(122=32.4 \%)$, and the sequencing of new genomes from the other phyla might reveal significant differences in genome size. Otherwise, significant $(P$-value $<0.5)$ differences were found in the number of KO functions were Ascomycota (2,666 $\pm 222 \mathrm{KO}$ functions) comprised of more

41 functions than Basidiomycota $(2,549 \pm 191)$ that, in turn, had more KO functions compared to 42 Blastocladiomycota (2,071 \pm 268$)$, Chytridiomycota $(2,001 \pm 276)$, Mucoromycota $(2,261 \pm 444)$ and 
Zoopagomycota (2,062 \pm 277$)$ (Figure 1b). Higher functional diversity in Ascomycota and Basidiomycota who belong to the subkingdom Dikarya may relate with their classification as higher fungi ${ }^{14}$ but requires the sequencing and full annotation of additional genomes especially from the other phyla for validation. In the 377 analyzed fungal species, the median of interspecies functional redundancy was found to be $0.03 \pm 0.41$ (Figure 1c). Interspecies redundancy describes the performance of one metabolic function by multiple coexisting and taxonomically distinct organisms ${ }^{15}$. In fact, most major biogeochemical reactions are driven by a limited set of metabolic pathways that are found in a variety of microbial clades ${ }^{16}$. Consistent, taxonomic diversity strongly correlated with functional diversity and many ectomycorrhizal fungal species with similar ecological effects co-occurred in the same community ${ }^{17}$ implying a high interspecies redundancy to mobilize nutrients from organic compounds ${ }^{18,19}$. Here, interspecies redundancy was either high as 1,592 KO functions were more than $90 \%$ of the fungi or low with 4,537 KO functions in less than $10 \%$ of the species; together describing $77.3 \%$ of all KO functions. Hence, functions appear to diverge into highly redundant across fungal species or unique to only a few. The median of intraspecies functional redundancy was 2.0 \pm 1.1 gene copies per KO functions (Figure 1d). Generally, low intraspecies redundancy could derive from different KO functions performing functionally similar processes. In fact, all malate dehydrogenases perform the same metabolic function but are annotated by different KO functions (K00024-K00029) due to their involvement in a variety of metabolic pathways. The maximum intraspecies redundancy was found for the ascomycete Coccidioides immitis RS that comprised 118 gene copies of the gustatory receptor (K08471) followed by 35 gene copies for the prolyl 4hydroxylase (K00472, EC 1.14.11.2) and 29 gene copies for the glutathione S-transferase (K00799, EC 2.5.1.18); all of which are functions with low interspecies but high intraspecies redundancy. Noteworthy, the intraspecies redundancy of $C$. immitis RS of $1.6 \pm 2.5$ was lower compared to the other fungi in the database, which could relate to a rather uncommon lifestyle with a higher share of unique but not essential functions. Indeed, comparative genomic analysis revealed that $C$. immitis is a primary pathogen of immunocompetent mammals ${ }^{20}$. Functions with high interspecies and high intraspecies redundancy included the yeast amino acid transporter (K16261) with 14.5 \pm 8.2 gene copies found in 351 of the 377 fungal species (93.1\%), the salicylate hydroxylase (K00480, EC 1.14.13.1) with 13.9 \pm 9.5 gene copies $(81.7 \%)$ and the glutathione S-transferase (K00799, EC 2.5.1.18) with $12.7 \pm 11.7$ gene copies (98.9\%). All of the above belong to the maintenance apparatus of the fungus, namely the transport of amino acids, the incorporation/reduction of oxygen by salicylate hydroxylase, and the detoxification of xenobiotic substrates by glutathione S-transferase. Hence, functions with high interspecies and high intraspecies redundancy are both widespread and essential to every fungus. Functions with high interspecies and low 
intraspecies redundancy were not found in the 377 genomes. Logically, there might not exist widespread functions that are not essential. Surprisingly and different to our hypothesis, the unsaturated model described the AC significantly better ( $P$-value $<2.2 \mathrm{E}-16$ ) than the saturated model with both lower Akaike information criterion (AIC) and residual sum of squares (Table 1). The unsaturated model is described by the maximum functional richness $f_{\max }$ of $4,716 \pm 18^{* * *}$ across the 377 fungal species with an accretion rate $A_{f}$ of $2.1 \pm 0.1^{* * *}$ per fungal species, consistent with the estimate of intraspecies functional redundancy (Figure 2a). However, the relationship did not plateau as indicated by the constant of the additive term $k$ that is $9.0 \pm 0.1^{* * *}$. As it was suggested by the interspecies redundancy, the better fit of the unsaturated model inferred the presence of two types of microbiome functions. On the one hand, widespread functions rapidly increase with the number of species and are ubiquitously abundant in every living fungi. In total, nine functions were found in all of the 377 fungal species. All of which are crucial to sustain life such as the ribose-phosphate pyrophosphokinase (K00948, EC 2.7.6.1) necessary for nucleotide synthesis, the citrate synthase (K01647, EC 2.3.3.1) of the TCA cycle or the superoxide dismutase (K045654, EC 1.15.1.1) that is an important antioxidant defense mechanism. The number of widespread functions is limited and the majority have been identified thus far amounting to, in total, 3,593 \pm 31 functions (with 3,534-3,654 as $95 \%$ confidence intervals); nearly half of all known functions as of today. Otherwise, roughly 4,300 functions are rare and increase at a much slower rate with an increasing number of species but require time and the evolution of "dead ends", i.e. species that were unable to evolve a particular function. The addition of more fungal genomes may increase the interspecies redundancy but it is questionable if a function only found in a few of the 377 fungal species can potentially be widespread amongst fungi. Given a species richness of 3.8 million fungi on Earth ${ }^{21}$ and assuming that the yet unknown fungal microbiome functions are indeed rare, the propagation of the unsaturated model predicted the total fungal microbiome functionality on Earth to be 42,373,186 $\pm 459,560$ (with 41,574,275-43,376,938 as $95 \%$ confidence intervals). This estimate was validated by using random subsets of $10,20,30,40,50,60$, 70,80 and $90 \%$ of all 377 fungal species, which yielded to a plateau of predicted functions when at least $70 \%$ of the species were used (Table 2). Consistent with the better fit of the unsaturated model from the parametric approach, the non-parametric estimator of functional richness Chao- 1 that assumes the existence of a maximum functional richness showed no plateau with increasing species richness of the lower bound estimate (Figure $\mathbf{2 b}$ ) implying that the given species richness is too little to reach a potential maximum of functionality. Admittedly, the exploration beyond the limits of the data is likely to be imprecise and the predictions of fungal microbiome functionality may differ once additional fungal 
genomes with potentially new functions have been added. However, as of today, our understanding of fungal microbiome functionality is likely limited to a marginal part of all functions.

Taken together, we suggest the presence of two types of fungal microbiome functions; widespread and rare functions. Our predictions revealed a potential for million more yet unknown rare functions that, logically, can only be unveiled by novel and more sophisticated methods. However, due to the vast amount of yet unknown functions, it is questionable if the relationship between taxonomy and function is in fact explained by an unsaturated model, if only two types of functions exist and if it is similar when different tools for the functional annotation are used. Noteworthy, the total bacterial microbiome functionality was not predicted as more than 70,000 bacterial genomes must be downloaded individually. At a rate of roughly a minute per genome, the estimated time to gather the information is more than 1,167 hours (or 146 work days). However, given the 100 million bacterial species on Earth ${ }^{22,23}$ together their lifestyle as micro-environment niche specialists ${ }^{24}$, the total bacterial microbiome functionality is likely to be much higher and hence, our understanding of the involvement of microbes in ecosystem functioning even lower.

\section{Materials and Methods}

\section{Metadata collection of the total known fungal microbiome functions}

To quantify the relationship between taxonomy and function, all genomes from taxonomically diverse fungal species (as taxonomic unit) were downloaded from the integrated microbial genomes and microbiomes (IMG) of the Joint Genome Institute (JGI) on August $7^{\text {th }} 2019$, containing the functional annotation from the level 3 of KEGG Orthology 8,9 (as functional unit) with counts of every gene per every genome. In total, the database comprised of 377 completely annotated fungal genomes with 7,926 KO functions. The gene counts and KO functions per fungal phylum and in all fungi were retrieved from the database. Interspecies redundancy was calculated as the number of KO functions covered by one randomly chosen species compared to the total number of functions in all species. Intraspecies redundancy or gene redundancy ${ }^{25}$ was estimated as average of genes per individual KO function in any one species.

\section{Accumulation curves $(A C)$}

Fungal species were randomly added in intervals of one up to the maximum species richness of 377 with 1,000 random permutations per step using the function specaccum from the R package vegan ${ }^{26}$. The $A C$ 
135 of the database permutation was then fitted to a saturated (Equation 1) and an unsaturated model

136 (Equation 2) with the critical point estimated by the term $3 A_{f}$ as previously described ${ }^{27}$. The fit of the 137 models was compared by analysis of variance (ANOVA) and Akaike Information Criterion (AIC) ${ }^{28}$ with a 138 penalty per parameter set to $k$ equals two. The total number of KO functions in fungi on Earth was 139 predicted using the global species richness estimate of 3.8 million fungi ${ }^{21}$ and the Monte Carlo simulation 140 of the function predictNLS in the R package propagate ${ }^{29}$. To validate the Michaelis-Menten approach, 141 random subsets of the 377 fungal species with different sizes were used to predict the total microbiome 142 functions as described before. In addition, the non-parametric estimation of the lower bound of functional 143 richness was calculated by Chao-1 again using random subsets of the 377 fungal species with different 144 sizes and 20 replicates each (Equation 3).

Eq. 1: Functional richness $=\frac{f_{\max } *[\text { Species richness }]}{A_{f}+[\text { Species richness }]}$

146 Eq. 2: Function richness $=\frac{f_{\text {max }} *[\text { [species richness }]}{A_{f}+[\text { Species richness }]}+k *[$ Species richness $]$

147 Eq. 3: Chao1 $=$ Functional richness $* \frac{a_{1}{ }^{2}}{2 a_{2}}$

148 Here, $f_{\max }$ is the maximum functional richness, $A_{f}$ the accretion rate of functions with an increasing number 149 of species and $k$ the constant of the additive term. Functions found only once or twice are indicated by $a_{1}$ 150 as singletons and $\mathrm{a}_{2}$ as doubletons, respectively. 


\section{References}

152 1. Hooper, D. U. et al. Effects of biodiversity on ecosystem functioning: A consensus of current 153 knowledge. Ecol. Monogr. (2005). doi:10.1890/04-0922

1542 2. Goldstein, P. Z. Functional ecosystems and biodiversity buzzwords. Conservation Biology (1999). doi:10.1046/j.1523-1739.1999.013002247.x

3. Jax, K. Function and 'functioning' in ecology: What does it mean? Oikos (2005). doi:10.1111/j.1600-0706.2005.13851.x

4. Lamont, B. B. Testing the Effect of Ecosystem Composition/Structure on Its Functioning. Oikos (1995). doi:10.2307/3545658

5. Woese, C. R., Kandler, O. \& Wheelis, M. L. Towards a natural system of organisms: proposal for the domains Archaea, Bacteria, and Eucarya. Proc. Natl. Acad. Sci. (1990).

6. HUBBELL, S. P. Neutral theory in community ecology and the hypothesis of functional equivalence. Funct. Ecol. (2005). doi:10.1111/j.0269-8463.2005.00965.x

7. Žifčáková, L. et al. Feed in summer, rest in winter: microbial carbon utilization in forest topsoil. Microbiome (2017). doi:10.1186/s40168-017-0340-0

8. Kanehisa, M., Sato, Y., Kawashima, M., Furumichi, M. \& Tanabe, M. KEGG as a reference resource for gene and protein annotation. Nucleic Acids Res. (2016). doi:10.1093/nar/gkv1070

9. Kanehisa, M., Sato, Y. \& Morishima, K. BlastKOALA and GhostKOALA: KEGG Tools for Functional

172 10. Pham, V. H. T. \& Kim, J. Cultivation of unculturable soil bacteria. Trends in Biotechnology (2012). doi:10.1016/j.tibtech.2012.05.007

174 11. Martiny, A. C. High proportions of bacteria are culturable across major biomes. ISME J. (2019).

175 12. Starke, R., Jehmlich, N. \& Bastida, F. Using proteins to study how microbes contribute to soil ecosystem services: The current state and future perspectives of soil metaproteomics. J. Proteomics (2018). doi:https://doi.org/10.1016/j.jprot.2018.11.011 
13. Gotelli, N. J. \& Colwell, R. K. Quantifying biodiversity: Procedures and pitfalls in the measurement and comparison of species richness. Ecology Letters (2001). doi:10.1046/j.14610248.2001.00230.x

14. Hibbett, D. S. et al. A higher-level phylogenetic classification of the Fungi. Mycol. Res. (2007). doi:10.1016/j.mycres.2007.03.004

15. S., L., L.W., P. \& M., D. Decoupling function and taxonomy in the global ocean microbiome. Science (80-. ). (2016). doi:10.1126/science.aaf4507 LK http://sfx.library.uu.nl/utrecht?sid=EMBASE\&issn=10959203\&id=doi:10.1126\%2Fscience.aaf450 7\&atitle=Decoupling+function+and+taxonomy+in+the+global+ocean+microbiome \&stitle=Science \&title=Science\&volume=353\&issue=6305\&spage=1272\&epage=1277\&aulast=Louca\&aufirst=Stili anos\&auinit=S.\&aufull=Louca+S.\&coden=SCIEA\&isbn=\&pages=12721277\&date=2016\&auinit1=S\&auinitm=

16. Falkowski, P. G., Fenchel, T. \& Delong, E. F. The microbial engines that drive earth's biogeochemical cycles. Science (2008). doi:10.1126/science.1153213

17. Rineau, F. \& Courty, P. E. Secreted enzymatic activities of ectomycorrhizal fungi as a case study of functional diversity and functional redundancy. in Annals of Forest Science (2011). doi:10.1007/s13595-010-0008-4

18. Bolger, T. The functional value of species biodiversity - A review. Biology and Environment (2001).

19. Walker, B., Kinzig, A. \& Langridge, J. Plant attribute diversity, resilience, and ecosystem function: The nature and significance of dominant and minor species. Ecosystems (1999). doi:10.1007/s100219900062

20. Sharpton, T. J. et al. Comparative genomic analyses of the human fungal pathogens Coccidioides and their relatives. Genome Res. (2009). doi:10.1101/gr.087551.108

21. Fungal Diversity Revisited: 2.2 to 3.8 Million Species. in The Fungal Kingdom (2017). doi:10.1128/microbiolspec.funk-0052-2016

22. Schloss, P. D. \& Handelsman, J. Toward a census of bacteria in soil. PLoS Comput. Biol. (2006). doi:10.1371/journal.pcbi.0020092

23. Curtis, T. P., Sloan, W. T. \& Scannell, J. W. Estimating prokaryotic diversity and its limits. Proc. 
Natl. Acad. Sci. (2002). doi:10.1073/pnas.142680199

207 24. Simard, S. W. et al. Mycorrhizal networks: Mechanisms, ecology and modelling. Fungal Biology

25. Pérez-Pérez, J. M., Candela, H. \& Micol, J. L. Understanding synergy in genetic interactions. Trends in Genetics (2009). doi:10.1016/j.tig.2009.06.004

211 26. Oksanen, J. et al. vegan: Community Ecology Package. R package version 2.5-2. CRAN R (2018).

212 doi:ISBN 0-387-95457-0

213 27. Čapek, P., Kotas, P., Manzoni, S. \& Šantrůčková, H. Drivers of phosphorus limitation across soil microbial communities. Funct. Ecol. (2016). doi:10.1111/1365-2435.12650

28. Bertrand, P. V., Sakamoto, Y., Ishiguro, M. \& Kitagawa, G. Akaike Information Criterion Statistics.

217 29. Spiess, A.-N. propagate: Propagation of Uncertainty. R Packag. version 1.0-6 (2018). 


\section{Figures}

220 Figure 1: Gene counts (a) and the number of KO functions (b) in fungal phyla given as average with 221 standard deviation. The number of fungal genomes per phylum is given in italic. Data followed by the 222 same letter is not statistically different according to the HSD-test ( $P$-value $>0.5$ ). Total share of KO 223 functions within fungi relative to the total number of fungal species in the database as interspecies 224 redundancy $(c)$ and the number of replicated KO functions within one fungal species in the database as 225 intraspecies redundancy (d).

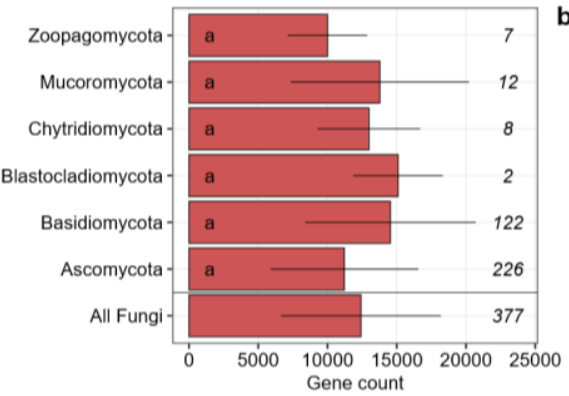

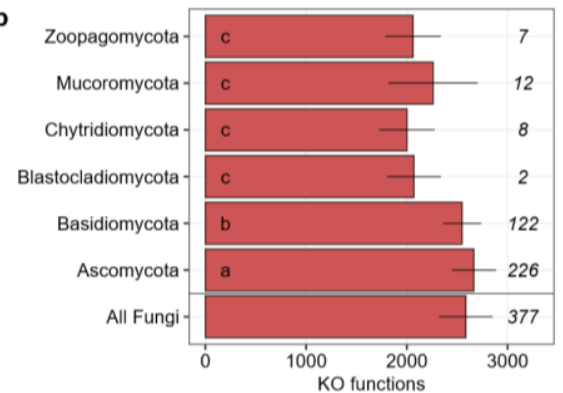

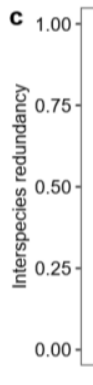

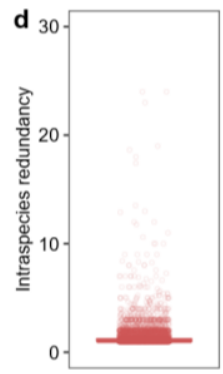


227 Figure 2: Parametric (a) and non-parametric (b) estimation of total functional richness. The unsaturated

228 model of the accumulation curves as grey points with error bars for the total known fungal microbiome

229 functions derived from the KO database by 1,000 random permutations for every one species richness

230 with $95 \%$ confidence intervals. The maximum functional richness is represented by $f_{\max }, A_{f}$ is the accretion

231 rate of functions with increasing number of species, and $k$ is the constant of the additive term. Significance

232 of the parameter estimates are indicated by asterisks (*** equals $\mathrm{P}<0.001$ ). The Chao- 1 was calculated

233 using 20 replicates shown in grey for every $10 \%$ of the total fungal species richness in the database starting

234 with two species.
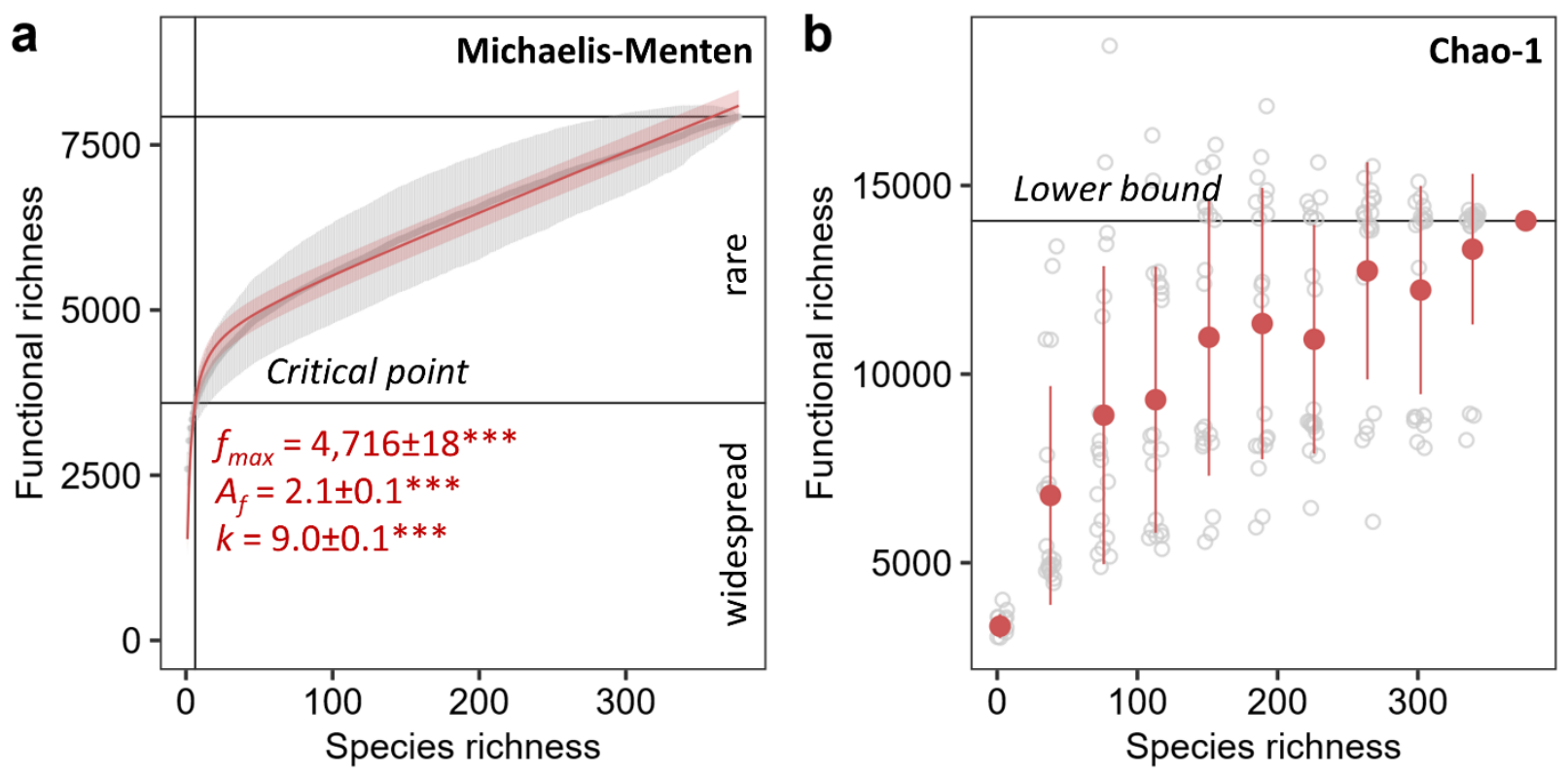


\section{Tables}

237 Table 1: The fit of the saturated (S) and the unsaturated model (US) of the accumulation curve indicated 238 by the Akaike's An Information Criterion (AIC) and residual sum of squares (Res. Sum Sq), the P-value that 239 describes the significant difference between the saturated and the unsaturated model, and the mean 240 prediction with standard deviation (SD) and 95\% confidence intervals (CI) at 3.8 million fungal species.

\begin{tabular}{llllllll}
\hline Model & AIC & Res. Sum Sq & P-value & Prediction & SD & Lower Cl & Higher Cl \\
\hline S & $5,843.751$ & $1.17 \mathrm{E}+08$ & & & & & \\
US & $4,693.567$ & 5514694 & $<2.2 \mathrm{E}-16$ & $42,373,186$ & 459,560 & $41,574,275$ & $43,376,938$ \\
\hline
\end{tabular}


242 Table 2: The mean prediction of total fungal microbiome functionality with standard deviation (SD) and

$24395 \%$ confidence intervals $(\mathrm{Cl})$ given a richness of 3.8 million fungal species on Earth estimated by the

244 Monte Carlo simulation when random subsamples of the 377 fungal species are used. The prediction

245 stabilizes at around 40 million functions with at least $70 \%$ of the fungal species.

\begin{tabular}{ccccc}
\hline Species (\%) & Prediction & SD & Lower Cl & Higher Cl \\
\hline $377(100)$ & $42,373,186$ & 459,560 & $41,574,275$ & $43,376,938$ \\
$339(90)$ & $37,575,880$ & 658,902 & $36,285,040$ & $38,868,781$ \\
$302(80)$ & $46,712,785$ & 732,903 & $45,274,956$ & $48,152,062$ \\
$264(70)$ & $38,412,964$ & 315,475 & $37,794,156$ & $39,031,118$ \\
$226(60)$ & $62,515,150$ & $2,085,421$ & $58,421,494$ & $66,607,622$ \\
$189(50)$ & $63,362,037$ & $1,645,403$ & $60,134,332$ & $66,591,118$ \\
$151(40)$ & $77,516,964$ & $1,427,367$ & $74,713,805$ & $80,321,171$ \\
$113(30)$ & $97,942,669$ & $7,026,524$ & $84,131,304$ & $111,757,847$ \\
$76(20)$ & $104,882,008$ & $11,188,137$ & $82,875,937$ & $126,831,401$ \\
$38(10)$ & $107,435,329$ & 3,650675 & $100,235,159$ & $114,636,999$ \\
\hline
\end{tabular}




\section{Acknowledgements}

248 RS thanks the Czech Science Foundation for the project 18-25706S. The authors thank Iñaki Odriozola for 249 the determination of the accumulation curves.

\section{Contributions}

251 RS designed the study. DM and RS performed the computational analysis. PC and RS modelled the data.

252 RS and NJ reviewed the analysis. The paper was written by RS, reviewed by all authors which approved 253 the final version of the manuscript.

\section{Competing interests}

255 The authors declare no competing financial and/or non-financial interests as defined by Nature Research, 256 or other interests that might be perceived to influence the results and/or discussion reported in this paper. 